\title{
Impaired Driving Associated with the Synthetic Cannabinoid 5F-ADB
}

\section{McCain $\mathrm{KR}^{1}$, Jones $\mathrm{JO}^{2}$, Chilbert $\mathrm{KT}^{3}$, Patton $\mathrm{AL}^{2}$, James $\mathrm{LP}^{4}$ and Moran $\mathrm{JH}^{\star 2,5}$}

${ }^{1}$ Department of Pharmacy Practice College of Pharmacy, University of Arkansas for Medical Sciences, Little Rock, AR, USA

${ }^{2}$ PinPoint Testing, LLC, Little Rock, AR, USA

${ }^{3}$ Baptist Health Medical Center - Little Rock, Baptist Health Dr., Little Rock, AR USA

${ }^{4}$ Department of Pediatrics College of Medicine, University of Arkansas for Medical Sciences, W Markham St \#550, Little Rock, AR, USA

${ }^{5}$ Department of Pharmacology \& Toxicology College of Medicine, University of Arkansas for Medical Sciences, Little Rock, AR, USA

*Corresponding author: Moran JH, PinPoint Testing, LLC, Little Rock, AR, USA, E-mail: jeff.moran@ pinpointtesting.com

Citation: McCain KR, Jones JO, Chilbert KT, Patton AL, James LP, et al. (2018) Impaired Driving Associated with the Synthetic Cannabinoid 5F-ADB. J Forensic Sci Criminol 6(1): 105

Received Date: July 13, 2018 Accepted Date: September 6, 2018 Published Date: September 7, 2018

\begin{abstract}
Synthetic marijuana compounds are more potent than $\Delta 9$-tetrahydrocannabinol ( $\Delta 9$-THC) and are known to produce a wide variety of clinical symptoms including cardiac toxicity, seizures, and death. Erratic driving by a $45 \mathrm{y} / \mathrm{o}$ male was witnessed in the fall of 2017 and roadside evaluation of the driver by the responding law enforcement officer concluded that the driver was intoxicated. Comprehensive analysis of the cigarettes by gas chromatography-mass spectrometry detected the synthetic cannabinoid 5-fluoro-ADB (5F-ADB or 5F-MDMB-PINACA). Validated forensic liquid chromatography-tandem mass spectrometry (LC-MS/MS) methods were used to detect the 5-fluoro ADB metabolite $7(26.37 \mathrm{ng} / \mathrm{mL})$ in the driver's blood sample. No other drugs were detected. This case report is one of the first to conclusively show that designer synthetic cannabinoids, commonly referred to as "K2" and "Spice", can significantly impair driving at relatively low concentrations.
\end{abstract}

Keywords: Forensic Science; Forensic Toxicology; Driving Under The Influence Of Drugs; Synthetic Cannabinoid; Spice; K2; Drug Recognition Expert; 5F-ADB; 5F-MDMB-PINACA; 5-Fluoro ADB

Abbreviations: LC-MS/MS-liquid chromatography tandem mass spectrometry; NPS-novel psychoactive substances; UNODC-United Nations Office on Drugs and Crime; $\Delta$ 9-THC- $\Delta$ 9-tetrahydrocannabinol; GC-MS-gas chromatography mass spectrometry

\section{Introduction}

The use of novel psychoactive substances (NPS) has grown in popularity throughout the past decade. As of December 2017, there were 779 NPS registered to the United Nations Office on Drugs and Crime (UNODC) Early Warning Advisory on NPS. Synthetic cannabinoids are the most common NPS and are a structurally diverse class with over 250 specific cannabinoids reported to be available [1]. Illegal laboratories make simple modifications to one or more structural components to mimic the effects of $\Delta 9$-tetrahydrocannabinol $(\Delta 9$-THC), evade laboratory detection, and challenge law enforcement, regulatory frameworks, and medical care providers [2]. These new designer drugs are commonly referred to as either "K2" or "Spice”. The following case report provides clinical and laboratory information from an impaired driver. Comprehensive analytical testing of blood samples and residual cigarettes confirmed the presence of the synthetic cannabinoid 5-fluoro-ADB (5F-ADB, 5F-MDMB-PINACA), an illicit compound that first appeared in the US in 2014. Knowledge of the pharmacokinetic/dynamic properties of 5F-ADB is important to future efforts to regulate this compound and other NPS.

\section{Case History}

A 45-year-old male was witnessed to drive his vehicle into oncoming traffic and swerve the vehicle into a roadside guard rail. When the responding law enforcement approached the vehicle, it remained running and engaged in reverse gear but was lodged against the railing. The driver was asleep, held a lighter in one hand and two blunt cigarettes were on the car seat. The driver responded to verbal stimuli, indicated that he was "high" and that the cigarettes "possibly" contained marijuana. He denied alcohol consumption. Law enforcement reported that the driver had bilateral horizontal and vertical nystagmus, no odor of intoxicants, 
and subsequently failed the Walk-and-Turn (unable to maintain heel to toe position, lost count while walking, and raised arms repeatedly) and One Leg Stand (put foot down and used hands to balance) field sobriety tests. He was arrested for operating a vehicle while intoxicated and transported to the hospital.

The driver's blood sample and the blunt cigarettes were collected and sent to a state-level ISO-17025 accredited laboratory. Plant material from the cigarettes was confirmed via gas chromatography mass spectrometry (GC-MS) to contain the synthetic cannabinoid derivative 5F-ADB. Alcohol testing of the blood sample was negative and immunoassay (Biochip Chemiluminescent Assay) screening was negative for the drugs/drug classes listed in Table 1.

\begin{tabular}{|c|c|}
\hline Test & Result \\
\hline Meprobamate & Negative \\
\hline Amphetamine & Negative \\
\hline Barbiturates & Negative \\
\hline Benzodiazepines & Negative \\
\hline Methadone & Negative \\
\hline Opiates & Negative \\
\hline Cocaine metabolites & Negative \\
\hline Zolpidem & Negative \\
\hline Cannabinoids & Negative \\
\hline Tramadol & Negative \\
\hline Fentanyl & Negative \\
\hline
\end{tabular}

Table 1: Immunoassay Screening Results

Due to the circumstances surrounding the case and the detection of a synthetic cannabinoid in the plant material, comprehensive toxicological testing of the blood sample was referenced to a private ISO-17025 accredited laboratory (PinPoint Testing, LLC, Little Rock, Arkansas). Previous liquid-chromatography tandem mass-spectrometry (LC-MS/MS) methods validated for detecting synthetic cannabinoids and other designer drugs were modified to detect 5F-ADB and 5F-ADB metabolite 7 (Cayman Chemical Company, Ann Arbor, MI) [3-5]. In brief, $0.25 \mathrm{~mL}$ of sample was pretreated with $0.25 \mathrm{~mL}$ of $0.5 \mathrm{M}$ ammonium hydroxide and extracted with two $0.90 \mathrm{~mL}$ fractions of ethyl acetate after loading on Biotage ISOLUTE ${ }^{\bullet}$ SLE+ Supported Liquid Extraction 96-well plates (Biotage, LLC, Charlotte, NC). The eluate was evaporated under a gentle stream of nitrogen to complete dryness and reconstituted in $100 \%$ methanol. The extracts were injected on a Phenomenex Kinetex phenyl-hexyl analytical LC column $(2.6 \mu \mathrm{m} 50 \times 4.6 \mathrm{~mm})$, ramping from $95 \%$ aqueous mobile phase ( $10 \mathrm{mM}$ ammonium formate) to $100 \%$ organic mobile phase $(0.1 \%$ formic acid in methanol) over 4 minutes and holding for 1 minute, before returning to initial conditions for a 2-minute column equilibration. 5F-ADB Metabolite 7 was confirmed in the blood sample at $26.37 \mathrm{ng} / \mathrm{mL}$ (Figure 1). Neither the parent drug or other confounding drugs were detected in the blood specimen.

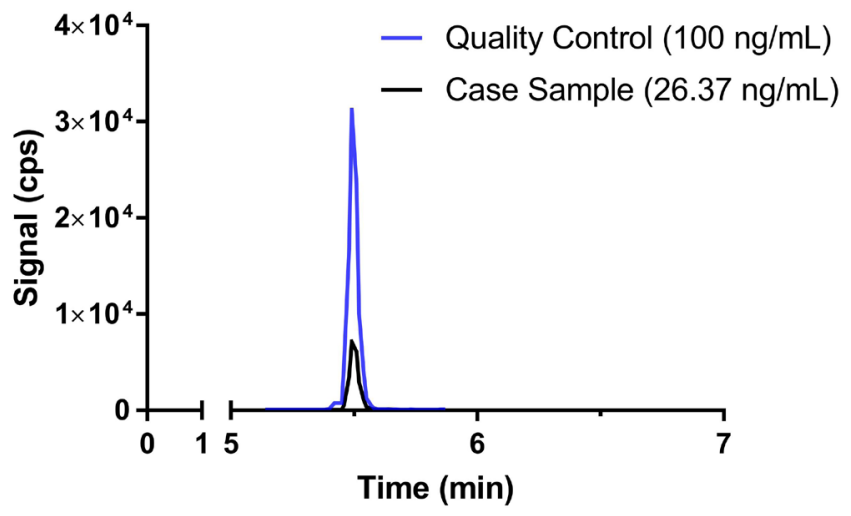

Figure 1: A representative LC-MS/MS chromatograph showing the Specific Reaction Monitoring experiment used to quantify the 5F-ADB Metabolite $7(364.2 \rightarrow 318.1 \mathrm{~m} / \mathrm{z})$ in the case sample (shown in black) overlaid with a quality control sample (shown in blue). Chromatography of all calibrators and quality control samples were similar throughout the analytical run. The LC-MS/MS analyses were performed on an Agilent 1260 quaternary liquid chromatography system (Santa Clara, CA) coupled to an Agilent 6420 tandem mass spectrometer (Santa Clara, CA).

\section{Discussion}

Synthetic cannabinoid users are attracted to synthetic marijuana due to drug potency and the expectation of euphoric effects. However, highly variable and serious adverse clinical effects including altered mental status, cardiotoxicity, seizures, acute kidney injury, hyperthermia, and death have been reported [2]. While these effects have been well documented, the degree to which users are impaired in the context of motor vehicle operation has not been thoroughly addressed in literature. 
The Drug Evaluation and Classification Program profile for marijuana includes well characterized eye findings (e.g., absence of horizontal or vertical nystagmus, the lack of convergence, normal to dilated pupils, normal to slowed pupillary reaction to light, injected conjunctivae), elevated heart rate, elevated blood pressure, normal to elevated temperature, body/eyelid tremors, confusion, relaxed inhibitions, poor performance on Standardized Field Sobriety Test and the odor of marijuana [6]. From the limited reports describing impaired driving linked to synthetic cannabinoid use, there appears to be variation from the profile utilized by Drug Recognition Experts to assess for marijuana associated impaired driving.

One retrospective study compared the differing effects of marijuana to synthetic cannabinoids within a population of arrested drivers and concluded there was greater impairment (e.g., confusion, disorientation, and incoherence) among those under the influence of synthetic cannabinoids, compared to those under the influence of marijuana [5]. A review of impaired driving cases in which AB-CHMINACA and AB-PINACA were detected in drivers' biologic samples found that clinical indicators identified by drug recognition experts differed from those classically associated with marijuana use. For example, impaired drivers were found to have the presence of horizontal and vertical nystagmus, the lack of convergence was not always present, lowered blood pressures, and infrequent presence of eyelid tremor [7]. Louis et al examined impaired driving cases associated with the use of XLR-11 and UR-144 [8]. They noted the slurred speech, lack of convergence, and body and eyelid tremors as the most consistent findings among 12 drivers examined by drug recognition experts [8]. However, physical signs and symptoms are not specific to synthetic cannabinoids, are highly variable, and may be caused by non-drug-related neurologic conditions.

$5 \mathrm{~F}-\mathrm{ADB}$ is an indazole synthetic cannabinoid receptor agonist that has greater potency than $\Delta 9$-THC and earlier generation synthetic cannabinoids [9]. The first human use reports involving 5F-ADB appeared in 2014. Common methods of use include smoking and vaping [10]. In laboratory-confirmed cases of use, symptoms associated with 5F-ADB include confusion, agitation, psychosis, altered consciousness, headache, dizziness, mydriasis, and vomiting [10,11]. On the basis of multiple analytically confirmed fatalities, 5F-ADB is regarded as one of the most dangerous synthetic cannabinoids [12].

The United States Drug Enforcement Administration provided notice of intent to temporary place 5F-ADB into schedule I of the U.S. Controlled Substance Act in December of 2016 [13]. Regulatory action often impacts several motivational factors including availability, price, and legal consequence. However, the ability of these drugs to evade detection in standard laboratory practices (as illustrated by this case report), regardless of regulations, remains as a primary motivational factor. Laboratories are challenged by the ever-changing drug market and the lack of specific analytical assays to accurately identify 5F-ADB and downstream metabolites. This report is the first to show the utility of using 5F-ADB Metabolite 7 as a biomarker for 5F-ADB use. The lack of the parent $5 \mathrm{~F}-\mathrm{ADB}$ in the blood specimen suggests that this synthetic cannabinoid has a relatively short half-life in vivo when inhaled, or that this cannabinoid is not stable in human blood. Future analysis of other impaired driving cases from 5F-ADB exposure will provide data needed to regulate this dangerous NPS.

\section{Study Approval}

The UAMS Institutional Review Board approved this study (UAMS IRB \# 206735).

\section{Acknowledgments}

The authors would like to express their gratitude to Kenneth Stecker and Kinga Canike of the Prosecuting Attorney's Association of Michigan (PAAM), the chemists and technicians of the Michigan State Police Toxicology Unit, and Thomas Hubbert of the Cass County Prosecutor's Office for their support on this project.

\section{Funding}

This work was supported by the National Institute of Health and National Institute for Drug Addiction [NIH/NIDA DA039143]

\section{Conflict of Interest Disclosure}

Amy Patton, Joseph Jones, and Jeffery Moran are employees and/or owners of PinPoint Testing, LLC.

\section{References}

1. United Nations Office on Drugs and Crime (2018) “Understanding the synthetic drug market: the NPS factor", Global SMART Update.

2. Ford BM, Tai S, Fantegrossi WE, Prather PL (2017) Synthetic Pot: Not Your Grandfather’s Marijuana. Trends Pharmacol Sci 38: 257-76.

3. Patton AL, Seely KA, Chimalakonda KC, Tran JP, Trass M, et al. (2013) Targeted metabolomic approach for assessing human synthetic cannabinoid exposure and pharmacology. Anal Chem 85: 9390-9.

4. Chimalakonda KC, Moran CL, Kennedy PD, Endres GW, Uzieblo A, et al. (2011) Solid-phase extraction and quantitative measurement of omega and omega-1 metabolites of JWH-018 and JWH-073 in human urine. Anal Chem 83: 6381-8.

5. Moran CL, Le VH, Chimalakonda KC, Smedley AL, Lackey FD, et al. (2011) Quantitative measurement of JWH-018 and JWH-073 metabolites excreted in human urine. Anal Chem 83: 4228-36.

6. Couper FJ, Logan BK (2004) Drugs and human performance fact sheets (DOT HS 809725). Washington DC: National Highway Traffic Safety Administration. 
7. Chase PB, Hawkins J, Mosier J, Jimenez E, Beesen K, et al (2016) Differential physiological and behavioral cues observed in individuals smoking botanical marijuana versus synthetic cannabinoid drugs. Clin Toxicol (Phila) 54: 14-9.

8. Louis A, Peterson BL, Couper FJ (2014) XLR-11 and UR-144 in Washington State and State of Alaska Driving Cases. J Anal Toxi 38: 563-8.

9. Banister SD, Longworth M, Kevin R, Sachdev S, Santiago M, et al (2016) Pharmacology of Valinate and tert-Leucinate Synthetic Cannabinoids 5F-AMBICA, 5F-AMB, 5F-ADB, AMB-FUBINACA, MDMB-FUBINACA, MDMB-CHMICA, and Their Analogues. ACS Chem Neurosci 7: 1241-54

10. WHO Expert Committee on Drug Dependence, thirty-ninth report (2018) Geneva: World Health Organization (WHO Technical Report Series, No 1009). License: CC BY-NC-SA 3.0 IGO.

11. Barcelo B, Pichini S, Lopez-Corominas V, Gomila I, Busardo FP, et al. (2017) Acute intoxication caused by synthetic cannabinoids 5F-ADB and MMB-2201: A case series. Forensic Sci Int 273: e10-4.

12. Hasegawa K, Wurita A, Minakata K, Gonmori K, Yamagishi I, et al. (2015) Identification and quantitation of 5-fluoro-ADB, one of the most dangerous synthetic cannabinoids, in the stomach contents and solid tissues of a human cadaver and in some herbal products. Forensic Toxi 33:112-21.

13. Fed Regist (2016) 81: 93595-9.

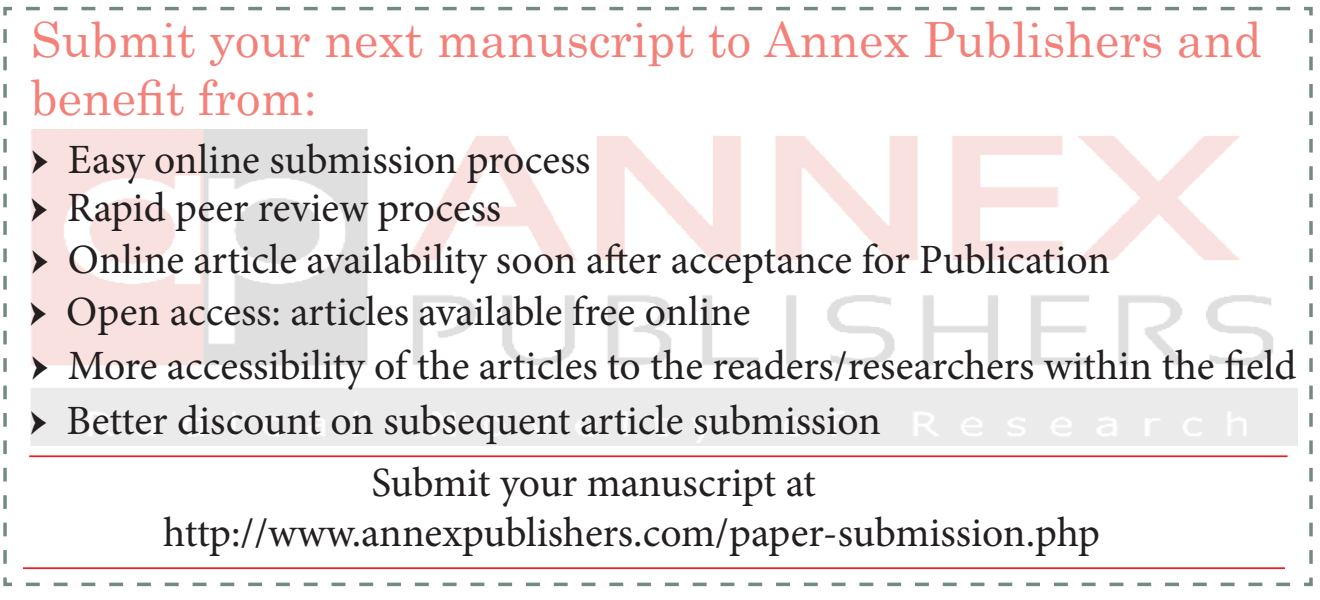

